\title{
La linguistique substrat du discours didactique : quand l'histoire nous parle au présent
}

\section{Henri Portine}

\section{OpenEdition}

Journals

Édition électronique

URL : http://journals.openedition.org/rdlc/1929

DOI : $10.4000 /$ rdlc. 1929

ISSN : 1958-5772

Éditeur

ACEDLE

Référence électronique

Henri Portine, «La linguistique substrat du discours didactique : quand l'histoire nous parle au présent », Recherches en didactique des langues et des cultures [En ligne], 6-2 | 2009, mis en ligne le 01 octobre 2009, consulté le 20 avril 2019. URL : http://journals.openedition.org/rdlc/1929 ; DOI : 10.4000/ rdlc. 1929

Ce document a été généré automatiquement le 20 avril 2019

\section{(1) $\Theta \Theta$}

Recherches en didactique des langues et des cultures is licensed under a Creative Commons AttributionNonCommercial-NoDerivatives 4.0 International License 


\title{
La linguistique substrat du discours didactique : quand l'histoire nous parle au présent
}

\author{
Henri Portine
}

\section{Introduction}

1 Nous allons d'abord nous interroger sur les objets que manipule le linguiste et sur le type de questionnements auxquels il est confronté ou qu'il envisage. Nous renversons ainsi l'ordre habituel qui consiste souvent à se demander ce dont la didactique a besoin et de voir alors ce que la linguistique peut lui apporter. Nous procédons ainsi parce que nous partons du postulat suivant: la didactique n'a rien à emprunter à la linguistique sauf l'essentiel: une vision de l'activité langagière et des objets qu'elle manipule, une méthodologie d'approche. Ces "préparatifs» nous permettront d'aborder ensuite la didactique des langues étrangères selon deux versions: une version chronologique d'abord (parce que les conditions et le contexte d'émergence est toujours important), une version tentant de faire le pont entre réflexion sur le langage et didactique ensuite. Nous n'espérons pas ainsi traiter exhaustivement des rapports entre linguistique et didactique des langues mais plutôt apporter des éclairages qui pourront conduire le lecteur à produire ses propres interrogations.

\section{Y a-t-il une base pour la notion de grammaire?}

2 Dès les premières études sur le langage, la question de l'objet à étudier a été une question difficile. Les mots étaient considérés comme des parties du logos, mais quelle est l'extension du logos ? La notion de partie appelle la notion de tout. Ce tout doit donc être défini pour que le statut de la partie soit lui-même défini. Historiquement, cette définition explicite $\mathrm{du}$ tout a pris plusieurs siècles pour aboutir - les grammairiens latins ayant traduit le 
terme logos des grammairiens grecs par onoma (ce qui fait perdre la dimension raisonnable du logos) - à la notion d'oratio perfecta que l'on considère comme la première manifestation de notre notion de phrase et dont on situe l'apparition au $14^{\text {ème }}$ siècle.

La notion de partie du logos ou de l'oratio était nécessaire non seulement parce que la notion de partie appelle la notion de tout mais aussi parce que l'objectif était de catégoriser lesdites parties $d u$ logos ou de l'oratio en noms, verbes, adjectifs, etc. Or toute catégorisation est la subdivision d'un ensemble qu'il faut nommer. Recourir à parties $d u$ logos puis à parties de l'oratio permettait de nommer cet ensemble sans grands risques.

La notion de phrase n'est donc pas une donnée basique mais une donnée ancillaire constituée au sein des études grammaticales. Pour faire de la grammaire, il faut des unités. Ces unités seront d'abord le mot, puis la phrase. On trouve encore la trace de cette mise en forme historique dans la formule " parties du discours ", peu utilisée aujourd'hui. Le terme « discours » ayant été adopté pour traduire le latin oratio, la formule « partie du discours" pouvait passer pour la traduction naturelle de "partie de l'oratio». Deux problèmes surgirent cependant: d'une part, le mot «discours » ayant pris le sens de "production étendue », la formule "partie du discours» devenait ambiguë (s'agissait-il d'un nom, d'un verbe, ou d'une introduction, d'une conclusion ?); d'autre part, le terme «discours » est resté vague. Il aurait été préférable de parler de "parties de phrase». Mais cette formule ne s'est jamais imposée. Au $18^{\mathrm{ème}}$, on trouve " parties de l'oraison », le latin oratio étant donc traduit par le français « oraison » mais ce dernier terme ayant pris une connotation religieuse ne pouvait perdurer dans cet usage.

On constate donc que l'on ne sait pas nommer l'objet de base sur lequel travaillent tous ceux qui ont affaire avec le langage. «Parties du discours » n'est plus utilisé. Le terme "mot» est inadéquat puisqu'il réfère à une unité séparée par deux blancs, unité graphique donc mais non unité grammaticale. Ainsi, « au fur et à mesure » comporte-t-il cinq mots alors qu'il se comporte comme un seul mot. Certes, on a inventé la notion de locution. Mais celle-ci est restée très imparfaite. On appelle «bien que " une locution parce qu'il s'agit de deux mots formant une conjonction unique et donc se comportant comme un mot. Cependant, on ne dira pas que « a mangé » est une locution verbale bien que ces deux mots forment un verbe unique.

6 Aujourd'hui, certains parleront de catégories grammaticales, d'autres dénieront à cette formulation le droit de désigner les noms, les verbes. Nous sommes donc dans une période de grand flou terminologique.

7 On pourrait penser qu'il n'y a là rien d'important. Utilisons mot, catégorie, peu importe. Mais on oublie alors que ce laisser-aller ne peut que compliquer l'intercompréhension. Gageons que cette difficulté à constituer une terminologie est l'une des causes du désintérêt de certains didacticiens de langue pour la linguistique. Les didacticiens peuvent, en effet, comprendre qu'il y ait des courants différents dans une discipline vivante, mais peut-on comprendre une incapacité à fonder une terminologie de base commune?

8 Si nous avons passé autant de temps sur un point qui peut paraitre mineur puisqu'il se traite en première année de linguistique ou de sciences du langage, c'est parce que, pour nous, cela met en évidence un double constat. Premier constat: il faudrait que les linguistes, en tant que corporation, s'entendent sur une terminologie de base non pas pour eux-mêmes mais pour la clarté des domaines d'application (mais pour cela il faut admettre qu'il y ait des applications, c'est-à-dire de l'intérêt des linguistes pour traiter du 
réel). Deuxième constat : ce ne sont pas les déclarations des linguistes qui importeront au didacticien, mais plutôt la démarche d'analyse et la façon d'envisager le langage.

Ce qui va donc être important, c'est ce que recouvre la notion de grammaire. Ajoutons, parce que cela va nous être utile, que la grammaire générative a mis en place un palier intermédiaire entre les items lexicaux et la phrase : celui de syntagme. La notion de syntagme existait déjà depuis plusieurs siècles mais ce qu'a fait la grammaire générative c'est dire qu'il fallait, parmi tous les syntagmes, en distinguer certains pour en faire des unités de niveau moyen. Nous avons donc aujourd'hui trois unités grammaticales : celle du mot (que l'on ne sait pas très bien désigner) ; celle du syntagme ; celle de la phrase. Les grammaires scolaires ont intégré la notion de phrase. Elles n'ont pas complètement intégré la notion de syntagme. On trouve, dans toutes les grammaires, la notion de syntagme nominal et quelques autres. Il faut remarquer que la grammaire générative utilise quatre syntagmes-unités ${ }^{1}$ : le syntagme nominal (le nom et ce qui le complète) et le syntagme adjectival (l'adjectif et ce qui le complète), qui ne posent pas de problèmes particuliers; le syntagme verbal (le verbe et ses compléments), assez bien assuré; le syntagme prépositionnel, qui est plus difficile à asseoir. Notons l'absence de syntagme adverbial, qui est généralement considéré comme analogue au syntagme adjectival. Lorsqu'on apprend une langue, on doit ainsi regrouper les mots en syntagmes puis en phrases.

\section{Trois notions : grammaire, langue, discours}

\subsection{La grammaire}

10 La notion première dans l'histoire de la linguistique est celle de grammaire. Il faut d'abord étudier comment les mots se regroupent en séquences (logos puis oratio). La grande différence entre la grammaire de l'Antiquité et celle d'aujourd'hui se trouve, nous venons de le voir, dans le fait que nous possédons aujourd'hui deux unités ensemblistes, celle de syntagme et celle de phrase.

11 Dans l'Antiquité et jusqu'au Moyen-Âge, on pratique la synthèse: la construction d'ensembles à partir d'unités de base. Dès que l'on a des unités ensemblistes (phrase puis syntagme) à sa disposition, on peut pratiquer l'analyse, la subdivision en parties.

12 La grammaire est ainsi un mouvement analytico-synthétique : on construit des ensembles syntagmatiques et phrastiques à partir des unités lexicales et des outils grammaticaux (synthèse); on divise les ensembles en unités plus petites jusqu'à l'obtention d'unités minimales (analyse). Cela suppose une propriété, la compositionnalité et la capacité à définir des relations.

Un mode d'analyse ${ }^{2}$ est compositionnel si le tout n'est que l'assemblage des parties. Pour la grammaire, la propriété de compositionnalité est très importante. Elle est d'ailleurs un gage de rigueur. Une phrase est et n'est que l'assemblage de ses syntagmes. Un syntagme est et n'est que l'assemblage d'unités lexicales et d'outils grammaticaux.

Les notions de phrase et de syntagme ne peuvent être que des assemblages. Dans son premier état, la grammaire générative ignorait la notion de fonction. Elle est aujourd'hui réintroduite de façon indirecte. La grammaire scolaire l'a toujours utilisée, parfois avec quelques problèmes terminologiques. L'analyse du syntagme adjectival «apte à conduire " ne peut se contenter de subdiviser ce syntagme en "apte» et en «à 
conduire ». Il faut ajouter que «à conduire» est le complément de «apte». Ce que l'on appelle fonction en grammaire scolaire est en fait une relation. Le terme «fonction » est d'ailleurs assez mal choisi. Une relation est clairement une relation entre deux objets alors que l'emploi de «fonction» ne met pas en évidence la nécessaire co-présence de deux objets. Prenons un exemple courant en classe de collège. L'enseignant demande d'analyser la fonction de "chat »ou de "le chat» dans le chat boit. L'élève répond « sujet ». Cela est faux (même si la lassitude de l'enseignant, ou un manque de formation ${ }^{3}$, le conduisent à ne pas corriger cette faute). Dire que «chat » est sujet c'est comme dire que «Jacques est oncle ». Ni l'un ni l'autre n'a la propriété « sujet » ou « oncle » dans sa substance. Alors que "chat» est substantiellement un nom (c'est sa catégorie d'appartenance) et que Jacques est substantiellement un homme (c'est sa catégorie d'appartenance). Comme Jacques est l'oncle d'Antoine, « chat » est le sujet de «boit » : ce sont deux relations.

Nous avons donc maintenant ce qui fonde la notion de grammaire: un mouvement analytico-synthétique, la compositionnalité et la solidarité relationnelle entre les composants d'une unité ensembliste.

\subsection{La langue}

Si la notion de grammaire a été au cœur de l'étude du langage jusqu'au $18^{\text {ème }}$ siècle, elle a été restreinte au statut de composante à partir du $19^{\text {ème }}$ siècle. Le terme "linguistique " apparait ainsi en 1820. Faisons un peu d'étymologie. Le nom "grammaire » est fondé sur le grec gramma (pluriel grammata), qui signifie « lettre(s)». Qu'est-ce qu'une lettre ? C'est l'unité de base d'un mot. Assembler des lettres donne un mot (synthèse); subdiviser un mot donne des lettres (analyse). Le nom "grammaire" porte donc en lui la notion de mouvement analytico- synthétique. En revanche, le nom « linguistique » est formé sur le latin lingua. Qu'est-ce qu'une langue ? Cette question appelle deux types de réponse.

La première réponse est sociopolitique. Lorsque le gouvernement français a voulu éradiquer les langues régionales, on a appelé patois ou dialecte (c'est-à-dire "variété régionale ») ces langues. C'était les disqualifier. Il en est ainsi parce que le terme langue a une connotation identitaire. On parle de la langue maternelle (en polonais, langue paternelle parce que c'est la langue de la patrie). Lacan a forgé le concept de lalangue (en un seul mot), ce qui met en évidence son unicité. Dans ses dialogues avec Mitsou Ronat, Chomsky répond ainsi à une question de Ronat " on dit par plaisanterie qu'une langue, c'est ce qui a une armée et une marine» (1977: 196). Cette phrase - qui a souvent été retenue, parfois attribuée à d'autres et, généralement, mal comprise - signifie que le concept de langue n'est pas un concept linguistique pour Chomsky (qui le remplace par les concepts de grammaire et de langage au sens mathématique), mais un concept politique. Chomsky précise d'ailleurs un peu plus loin que "les questions de langues sont toujours liées à celles $d u$ pouvoir ». Il aurait pu ajouter « du territoire».

La seconde réponse est technique. Une langue, c'est un triplet < lexique - outils grammaticaux - règles grammaticales >.

Le lexique est l'ensemble des lexèmes, c'est-à-dire du matériel lexical qui permet de désigner. On remarquera son extension possible : ainsi «canard» en français a-t-il pu en arriver jusqu'à désigner un journal. Ces extensions se forment selon des procédés divers et les relations sous-jacentes demeurent ou non repérables (un enseignant peut expliquer «canard " pour désigner le sucre dans un verre d'alcool en mimant le bec du canard qui 
plonge mais il sera bien en peine de justifier de façon simple la valeur «journal» de « canard»).

Les outils grammaticaux sont les termes qui permettent d'articuler entre eux les lexèmes et / ou les groupes déjà formés. On voit ainsi apparaître deux domaines distincts : celui des lexèmes (autonomes, noms et adjectifs notamment, ou non autonomes, les affixes), qui forment le matériel signifiant de base ; celui des termes (ou « outils ", « instruments ", la terminologie n'est pas fixée) grammaticaux (autonomes, déterminants, prépositions, conjonctions, ou non autonomes, les flexions, notamment), qui permettent de structurer des ensembles construits et hiérarchisés. Ces deux domaines comportent, comme on vient de le voir, leurs unités autonomes (noms, prépositions, etc.) et leurs unités non autonomes (affixes, flexions). Restent les éléments à cheval sur ces deux domaines : les verbes relèvent à la fois des lexèmes et des outils grammaticaux (ils sont signifiants et ont un pouvoir structurant très fort, ne sont que semi-autonomes puisqu'ils nécessitent généralement la présence obligatoire d'une flexion ${ }^{5}$ ); les adverbes se répartissent en adverbes lexématiques ("difficilement», par exemple), en adverbes grammaticaux (" très », par exemple) et en adverbes mixtes (" heureusement » est lexématique dans il a heureusement traité le problème et grammatical dans heureusement qu'il a réussi).

Les règles grammaticales - c'est-à-dire la grammaire proprement dite - sont les règles qui permettent d'assembler des mots et des flexions, des mots pour former des syntagmes, des syntagmes pour former des phrases $^{6}$, mais aussi de subdiviser les ensembles ainsi obtenus. La grammaire étudie les contraintes de bonne formation (syntaxe) et les valeurs associées à ces compositions / décompositions (sémantique).

Le passage de grammaire à linguistique a donc eu deux conséquences. La première a été de mettre la grammaire à sa place, celle d'une composante, et d'ajouter le champ lexical comme domaine d'étude à part entière. S'est alors posée la question de l'emploi des mots et de leurs relations. Une véritable sémantique lexicale a commencé à se constituer. Cela veut dire : ne plus considérer les lexèmes seulement dans leur pouvoir de désignation mais aussi comme les pièces d'un ensemble qui décrit le monde, éventuellement à partir d'un point de vue culturellement marqué. Le lexique n'est plus alors seulement l'ensemble des items qui remplissent des «trous » dans les structures grammaticales et qui imposent des relations entre éléments (la séquence la pierre court n'est pas une phrase parce que « pierre » désigne un inanimé et « court » réclame un sujet animé), mais un véritable domaine à part entière ${ }^{7}$.

23 La seconde conséquence est l'augmentation considérable du territoire à prendre en compte. Puisqu'une langue a une histoire, puisqu'une langue a des aspects sociopolitiques, il faut étudier les relations entre les langues, les effets de voisinage et de parenté, mais aussi les relations au sein des langues (coexistence de dialectes, hiérarchie de ces dialectes). Puisqu'une langue est un objet complexe (lexique et grammaire), il faut étudier la mise en œuvre de cette structure, d'où l'apparition de la pragmatique - c'està-dire de l'analyse des usages en situation - comme composante de la linguistique. Ainsi, si une langue se compose d'un lexique et d'une grammaire, la linguistique se composerat-elle d'une morphologie, d'une syntaxe et d'une sémantique (déjà présentes) mais aussi d'une pragmatique. L'éclosion d'une pragmatique devait entraîner la prise en compte de phénomènes de mobilisation de la langue dans une production, comme les actes de langage ou l'énonciation. 


\subsection{Le discours}

La notion de discours se heurte d'emblée à deux difficultés. La première est celle du rapport au monologue et au dialogue. La seconde est celle de la coprésence de deux termes, ceux de discours et de texte. Traitons d'abord la première. Il faut s'entendre sur les notions de monologue et de dialogue. Mikhail Bakhtine a mis en évidence le fait que toute prise de parole s'inscrit dans un ensemble de paroles produites ou productibles. C'est ce qu'on appelle l'aspect dialogique de la parole. Ce dialogisme se retrouve aussi bien à l'oral qu'à l'écrit. Bakhtine, dans ses études littéraires, oppose Tolstoï, auteur monologique, à Dostoïevski, auteur dialogique. Les héros de Tolstoï sont d'une seule pièce et portent la vérité de l'auteur tandis que ceux de Dostoïevski sont plus complexes et assument des paroles différentes. Nous avons tous le sentiment que, lorsque nous parlons, nos paroles font écho à d'autres paroles et que nous formulons nos propos en fonction de nos interlocuteurs. Appelons dialogisme basique cet aspect de notre parole. Il existe néanmoins deux cas : celui où nous exposons nos positions par de longues prises de parole et celui où nous construisons nos positions dans une interaction vivante. Afin d'éviter les recoupements avec l'opposition «monologique / dialogique », appelons « monologal » le discours dans une longue prise de parole et « dialogal » le discours dans une interaction vivante. L'écrit sera généralement monologal : nous exposons notre point de vue, nous rapportons celui d'autrui. L'oral peut être monologal (cas des discours devant un auditoire, des plaidoiries des avocats, des prises de position devant des amis, etc.) ou dialogal (interaction). On aura donc des discours écrits, des discours oraux monologaux et des discours oraux dialogaux.

La seconde difficulté à laquelle se heurte la notion de discours est celle de son rapport au texte. S'agit-il de deux synonymes? Sinon, où situerons-nous la différence? Pour la problématique de l'analyse de discours, nous renverrons aux deux ouvrages de Maingueneau (1976 et 1987) qui, bien qu'anciens, conservent leur actualité. Nous nous limiterons à deux remarques.

La notion de discours porte en elle la notion d'inachèvement tandis que la notion de texte paraît plus adaptée aux productions achevées. C'est pourquoi nous situerons le discours dans la production et le texte dans le résultat. On évalue des textes. On apprend à produire du discours. Nous conviendrons que cette distinction peut sembler un peu rapide et surtout assez approximative. Mais nous lui fixerons ici un but uniquement pédagogique : la distinction entre ce que l'on évalue et la capacité à produire que nous visons chez l'apprenant.

On a, pendant une vingtaine d'années, prôné une typologie des textes. Aujourd'hui, cette typologie des textes est, non pas abandonnée, mais mise de côté parce que trop tranchante et insuffisamment en prise avec les conditions de production. Cette notion de types de textes est en train de laisser la place à la notion de genre discursif. La notion de genre discursif présente deux grandes différences avec celle de types de textes. Elle n'est pas close. À l'époque de la prédominance des types de textes, une question récurrente était : combien de types? Certains se sont affrontés sur cette question: y a-t-il un type informatif ou non, étant donné que toute production s'adresse à quelqu'un et est donc fonction d'un auditoire, ce qui tendrait à faire du caractère informatif une composante d'un type explicatif? La notion de genre discursif se veut ouverte : on peut concevoir un genre « dissertation scolaire », un genre " épistolaire », etc., les conditions de production 
et de réception jouant un grand rôle dans la caractérisation de chaque genre. Cette notion de genre renoue avec la rhétorique aristotélicienne. La notion de formation discursive développée par Michel Pêcheux devrait être, elle aussi, réinterrogée dans ce cadre.

Pour terminer sur la question du discours, remarquons que la notion de corpus (qui est beaucoup plus ancienne) se développe depuis 1990. À l'origine de ce développement, il y a l'apparition de langages de balisage de données et la possibilité de traiter de grands ensembles grâce à des mémoires informatiques de très grande capacité. S'est ainsi constituée une linguistique de corpus qui se transforme en englobant d'autres questionnements. On trouve aussi de plus en plus de travaux sur son utilisation en classe de langue.

\section{Pourquoi l'histoire nous parle-t-elle au présent en didactique?}

Ce que nous venons de décrire, qui correspond grosso modo à une partie du contenu des études de première année en sciences du langage ou de linguistique en langues vivantes, représente en fait un ensemble de questions et, en même temps, un aperçu sur la genèse de la linguistique ${ }^{8}$. Comme nous l'avons vu, encore aujourd'hui, la linguistique a des faiblesses terminologiques qui, pour partie, correspondent à ses avancées actuelles et, pour partie, forment des traces de son histoire.

Il y a un autre aspect qui n'est pas négligeable. Le linguiste en tant que linguiste, et non en tant qu'enseignant de la linguistique, a tout son temps (sauf s'il veut être bien évalué et progresser dans sa carrière). La science avance à son rythme. Elle pose des questions, essaie de répondre à ces questions, ce qui entraîne de nouvelles questions ou montre que la question initiale était mal posée. En revanche, l'enseignant de langue agit toujours dans la précipitation. Il doit préparer ses cours pour le lendemain, répondre aux questions des apprenants9. De plus, il n'y a pas de traduction immédiate du savoir des linguistes en savoir- faire pédagogique. C'est un savoir utile mais ce n'est pas un savoir procéduralisable.

31 L'action en didactique des langues a donc plus de rapport avec le questionnement du linguiste et avec la genèse de la linguistique qu'avec les résultats à un instant $t$ de cette discipline. C'est pourquoi nous dirons que la genèse de la linguistique, dans sa recherche des bonnes questions et des bonnes façons d'analyser, mais aussi dans sa recherche des bons objets et de la bonne façon de les nommer, est plus importante pour la didactique des langues que l'ensemble des résultats obtenus par telle ou telle école. Cela appelle toutefois deux remarques.

La première remarque porte sur ce que l'on entend par genèse de la linguistique. Une branche de la linguistique s'est constituée sous la dénomination "histoire de la linguistique ». Ce n'est pas ce domaine que nous visons, même s'il nous fournit des éléments. Faire l'histoire de la linguistique, c'est aller bien au-delà de ce dont nous avons besoin. Par "genèse de la linguistique ", nous entendrons conscience de la formulation des questions, évolution des questionnements et regard épistémologique.

La seconde remarque porte sur le rapport aux résultats. Il existe des savoirs de base. Or ces savoirs de base, nous ne les avons pas en tant que locuteurs. Il en est ainsi parce qu'il y a une idéologie de la langue à parler pour être un locuteur digne de considération. Mais, dans les faits, lorsqu'ils se prêtent au jeu de l'enregistrement non contrôlé par eux, les 
locuteurs sont toujours surpris par ce qui constitue le matériau effectif de leur parole. Ces savoirs de base constituent un corpus qui comporte des savoirs sur la prosodie, des savoirs sur la syntaxe de l'oral, mais aussi sur la façon dont se constituent les discours oraux et écrits.

Il faut aussi avoir conscience d'un dernier point. La linguistique est prise entre deux pôles : le pôle formel et le pôle anthropologique. Une langue c'est à la fois un outil qui permet de produire de la science et un outil qui permet de se situer dans un échange, de coopérer dans la réalisation de tâches, de négocier en étant de bonne ou de mauvaise foi. La linguistique présente des analogies. Elle peut décider de se restreindre à l'étude des composantes phrastiques; elle tendra alors à être formelle. Elle peut décider d'investiguer les comportements langagiers humains dans leur complexité; elle tendra alors vers un traitement beaucoup plus anthropologique. Ni l'une ni l'autre de ces deux tendances ne forme la bonne linguistique; elles ont toutes les deux leur légitimité. On remarquera cependant que la didactique des langues a plus d'affinités avec une linguistique à orientation anthropologique. C'est pourquoi la sociolinguistique ou une linguistique de l'énonciation paraissent plus adéquate au traitement des tâches auxquelles sont confrontés à la fois l'enseignant et l'apprenant de langue.

\subsection{Chronologie et focalisations thématiques}

Nous ne prétendons pas faire ici œuvre d'historien de la didactique des langues. D'ailleurs, nous ne nous intéresserons qu'à des méthodologies datant d'après 1880 et nous ne prétendrons pas convoquer ici toutes les méthodologies. L'histoire de la didactique des langues - comme l'histoire de la linguistique - tend à devenir une discipline en soi. Remarquons à titre d'exemple que, pendant la première moitié du $16^{\text {ème }}$ siècle, paraît Lesclarcissement de la langue francoyse de John Palsgrave destiné au lecteur anglophone.

Comme ce fut le cas pour l'enseignement de l'histoire, on peut s'interroger sur ce qui doit être premier : la chronologie ou les thématiques (c'est-à-dire ce que l'on traite) qui font l'objet d'une focalisation? Chaque procédure a ses inconvénients. Se centrer uniquement sur la chronologie revient à voir la genèse comme moments de naissances et de reprises, ce qui aplatit les thématiques en cause. Se centrer uniquement sur les thématiques, c'est nier l'inscription des méthodologies dans des contextes historiquement définis. Toutefois, l'approche thématique porte en elle des éléments de chronologie puisqu'un thème (comme le traitement de l'écrit, le traitement de la relation à un autre apprenant, le traitement de la relation à la grammaire, etc.) est aussi lié à des conditions d'émergence.

\section{2. Éléments de chronologie}

Notre approche sera à la fois chronologique et thématique. Mais, afin d'éviter les effets pervers de l'approche thématique ${ }^{10}$, donnons d'abord des indications chronologiques, rapides donc un peu approximatives et très loin d'être exhaustives (voir Besse, 1985).

Années 1880-1890: François Gouin propose une méthode naturelle qui consiste principalement à gloser le déroulement d'actions, par exemple en décomposant l'action de sortir (« aller jusqu'à la porte, saisir la poignée », etc.). Dans le même temps, se met en place la méthode directe fondée principalement sur le refus de toute production en langue maternelle et sur l'emploi de la conversation. 

Basic English par Ogden. L'idée est de pouvoir dire le plus de choses possibles, y compris à l'aide de périphrases.

40 À la suite de la deuxième guerre mondiale, pour l'anglais, se met en place la méthode audio-orale qui est fondée sur une approche behavioriste des apprentissages (approche stimulus / réponse); les énoncés sont courts, plutôt quotidiens et la visée est principalement automatisante.

41 - Pour le français, la deuxième guerre mondiale marque la fin de sa domination culturelle et diplomatique au profit de l'anglais ; on assiste alors à la mise en place de la méthodologie Sgav (structuro-globale audio-visuelle), qui est une méthode directe centrée sur des dialogues fabriqués dans une langue très normée, reposant sur une forte prééminence de l'oral (le manuel de l'élève ne comporte que des images et l'écrit est le résultat d'un passage à l'écrit) et postulant un apprentissage en cours intensifs. On considère que l'apprentissage a lieu «en situation" par le recours à des images fixes supports des explications et du travail; chaque leçon se déroule en cinq phases (présentation, explication, mémorisation - correction phonétique, exploitation structurée, exploitation libre); on va ainsi d'une exigence de mécanicité à la recherche d'une créativité minimale ; la référence est la méthode Voix et images de France, qui paraît en 1962 et qui est fondée sur Le Français fondamental (inventaire lexical); pendant plusieurs années, la méthode ne sera vendue qu'accompagnée d'un stage de formation. On constate quelques évolutions dans la méthode De vive voix (1971), comme l'abandon de métadonnées dans les images (par exemple, une bulle avec «? "pour indiquer que la séquence orale liée à l'image est une question), comme moins d'images par séquence orale, comme une introduction de l'écrit effectuée plus rapidement.

La fin des années 1960 joue un rôle pour l'anglais langue étrangère, la forte immigration de locuteurs plus ou moins anglophones exige un travail sur le discours scolaire émanant de l'institution, des enseignants et des élèves.

- Vers 1973 émerge, en anglais langue étrangère, la notion d'approche communicative qui implique la prise en compte des variétés de langue, la mise en avant des interactions et le recours à des actes de langage ou de parole (speech acts) et le travail en binômes ( pairwork).

44 Au cours des années 1970, le Conseil de l'Europe publie The Threshold Level et Un niveauseuil; tous les deux cherchent à définir les matériaux nécessaires pour construire un niveau seuil permettant la circulation des migrants en Europe ; ils popularisent les actes de parole (ou de langage); la version française s'essaie à une grammaire notionnelle fondée sur le sens.

45 lui reproche son rôle de carcan bridant toute expression libre, reposant sur une langue artificielle, interdisant tout recours à des expressions un peu longues. Cette période de remise en cause sera suivie d'une période d'essais ; la critique sur le manque d'expression libre donnera lieu au recours à des exercices de créativité; la critique du caractère artificiel de la langue entraînera le recours à des documents dits " authentiques ", à une distinction entre discours quotidien et discours surveillé, à une prise en compte des discours spécifiques; la critique sur l'absence d'expressions un peu longues aura pour conséquence une meilleure prise en compte de l'écrit, l'élaboration d'une approche globale des textes écrits, un accent moins prononcé sur le dialogue, la prédominance de 
l'expression sur les apprentissages grammaticaux. On verra ainsi surgir une didactique fonctionnelle (c'est- à-dire orientée vers les discours spécialisés, à ne pas confondre avec functional qui désigne le recours aux actes de langage), une didactique des documents authentiques, une approche communicative, une approche notionnelle (qui se veut guidée non par la forme mais par le sens).

- À la fin des années 1970, en France, un groupe d'anglicistes de l'Institut d'anglais Charles V (université Paris 7), élabore une méthode d'anglais, Behind the words (Charlirelle, 1975-1980) qui essaie de dynamiser la notion d'énonciation pour les classes de collège ; la notion d'énonciation utilisée était liée à la notion d'opération cognitive et langagière.

47 Toujours à la fin des années 1970, se développe l'idée qu'il n'y a pas de "passage à l'écrit» (ce que prétendait le Sgav) mais un parallélisme entre production écrite et production orale et que chaque type de production a ses règles propres; de ce fait, se met en place une véritable théorie de la production écrite dont le point culminant sera le schéma de Hayes et Flower, tandis que se constitue une conception globale de l'approche des textes qui vise, non une lecture globale, mais une compétence de lecture fondée sur la primauté des opérations de haut niveau et donc sur une approche d'abord globalisante et donnant lieu ensuite à analyse (rapport haut niveau / bas niveau théorisé par P. Lindsay, D. A. Norman et D. E. Rumelhart au milieu des années 1970).

48 Au cours des années 1980, en France, Daniel Gaonac'h fait des rapprochements entre approche communicative et approche cognitive; mais ce mouvement a été peu suivi; dans le même temps, les concepteurs de méthodes avaient tendance à réintroduire des exercices grammaticaux d'inspiration souvent structurale.

49 La fin des années 1990 voit se développer une pédagogie fondée sur la réalisation de tâches (Rod Ellis, 2006 ; Cadre européen commun de référence, 2001), ce task based learning prolonge le task based teaching de N. S. Prabhu dans le cadre du Bangalore Project à la fin des années 1980. Avec Ellis, se met en place une véritable étude du processus SLA (second language acquisition), déjà profilée par Krashen en 1975 puis par les études sur l'interaction en classe de langue à partir de la fin des années 1980.

50 - À la fin des années 1990, une réflexion sur le plurilinguisme (comme paramètre d'une citoyenneté européenne et comme aptitude à l'apprentissage d'autres langues et à l'intercompréhension) se renforce; il s'agit d'une conception autant politique que pédagogique avec des implications curriculaires éventuelles importantes.

51 Le début des années 2000 voit l'émergence d'un grand intérêt pour les corpus et leur utilisation en classe de langues (O'Keefe et al., 2007) ; c'est l'une des conséquences de la constitution d'une «linguistique de corpus » au cours des années 1990, fondée sur les possibilités qu'offre Internet, dont la genèse se fait des années 1960 (Arpanet, réseau US d'abord à finalité militaire) à l'apparition du web en 1989-1990 (Internet repose sur des protocoles partagés, comme TCP/IP, http, et des composantes qui furent d'abord dissociées puis intégrées).

52 Depuis 2005, les notions de coaching et différents types de relation one-to-one sont à l'étude, ce qui devrait entrainer la structuration de tout un ensemble de types de relations pédagogiques finalisées.

53 Remarque sur les technologies de l'information et de la communication (TIC): longtemps qualifiées de nouvelles (NTE, NTIC, etc.) - ce qui relevait plus de la publicité que de la caractérisation objective -, les TIC ont transformé radicalement la question de 
l'EAO (enseignement assisté par ordinateur) ou du CAL (computer assisted learning) en faisant passer de l'attitude qui consiste à ramener la problématique des apprentissages aux potentialités de l'outil informatique à l'attitude qui cherche à mettre les réseaux informatiques au service de l'apprentissage; aujourd'hui, il est courant d'utiliser une plate-forme de formation (learning management system) et l'on essaie de l'adapter aux styles d'apprentissage, de lui adjoindre des activités complémentaires (ingénierie linguistique) et de passer au stade $\mathrm{du}$ « web 2 » (possibilité de catégoriser l'information).

\subsection{Une approche thématique}

Énonçons deux principes.

- Une méthodologie ne peut sélectionner purement et simplement un aspect des apprentissages; toute méthodologie essaie de se prononcer sur tous les aspects des apprentissages linguistiques; par exemple, la préface de Voix et images de France (cf. cidessus) précise (page X) : «l'image fixe - ou plutôt la succession d'images fixes - a été préférée, du moins pour l'instant, à l'image cinématographique, pour des raisons pratiques [...] et pour des raisons théoriques: l'image devant toujours précéder de quelques secondes la phrase sonore, et ne s'effacer qu'après elle, il faut que l'attention de la classe se concentre au maximum afin que situation et langage soient parfaitement associés. [...] l'image cinématographique [...] est en perpétuel devenir $»$.

- Une méthodologie ne peut pour autant traiter de tous les aspects des apprentissages ; il faut donc procéder à une ou des focalisations thématiques ; cela signifie que tel thème sera prédominant tandis que tel autre sera secondaire ou mineur ; c'est le cas du flux sonore dans la citation qui précède : un apprenant doit à la fois structurer correctement ses énoncés (correction ou accuracy) et se préparer à agir en temps réel (habileté langagière ou fluency) mais il ne peut faire les deux en même temps ; thématiquement, il faut donc subordonner l'un à l'autre; Voix et images de France met au premier plan la correction et au second l'habileté langagière.

Apprendre une langue est un acte complexe et long. C'est cette complexité qui explique la nécessité de focalisations thématiques. Enseigner une langue, c'est choisir ce que l'on doit travailler avec les apprenants; s'en remettre à une méthode, c'est abandonner ce choix aux auteurs de la méthode utilisée.

57 Nous allons proposer quelques focalisations thématiques. Nous les regrouperons en deux ensembles, celles qui portent sur un rapport et celles qui portent sur un domaine.

\subsection{Focalisations thématiques sur un rapport}

\subsubsection{Le rapport apprenant - monde}

Les méthodologies qui procèdent ainsi visent à réinscrire la classe dans le monde, réinscrire parce que l'institution l'a « désinscrite » du monde. Cette réinscription ne tend pas à annuler la désinscription mais à construire un rapport nouveau entre la classe et le monde parce qu'il faut " protéger » la classe des aspects brutaux du monde extérieur ${ }^{11}$. Le type même de cette réinscription est la correspondance scolaire dans la pédagogie Freinet. Elle a une temporalité : la classe prépare cette correspondance, elle travaille à partir d'une image de son auditoire, elle construit son écrit, y revenant à plusieurs reprises. Cette correspondance est aussi sociale: on écrit collectivement et 
individuellement, les écrits sont validés collectivement. Cette correspondance scolaire est l'une des rares manifestations de la focalisation sur le rapport au monde. On en trouve une autre dans les enquêtes que l'on mène dans les écoles Montessori. Il ne s'agit jamais, bien évidemment, de transformer l'élève en migrant soumis à un "apprentissage naturel ", même si ces pédagogies se veulent souvent naturelles. Il y a toujours un travail de reformulation et de réappropriation au sein de la classe.

\subsubsection{Le rapport apprenant - apprenant (modélisation du monde)}

59 Ce type de focalisation thématique cherche à faire un "petit monde » de la classe. Il ne s'agit donc pas de réinscrire la classe dans le monde mais d'inviter le monde dans la classe qui devient ainsi une sorte de "modèle du monde ». Les jeux de rôle s'y essaient mais à des degrés différents. Ainsi, les jeux de rôle de la suggestopédie n'ont-ils rien à voir avec cette focalisation puisque les élèves apprennent leurs rôles, pré-écrits, par cœur. En revanche, des jeux de rôle peu préparés peuvent être considérés comme des essais de modélisation du monde extérieur.

Les simulations globales, dues à Francis Debyser (à partir d'une réflexion sur La vie mode d'emploi de Georges Perec) et reprises par d'autres membres du Belc (Jean-Marc Caré, Francis Yaiche, Patrick Charrière, etc.), illustrent bien cette tentative de modéliser le monde. Il s'agit de construire, pas à pas, avec les élèves, un petit univers : ce fut d'abord un immeuble, puis le peuplement d'une île déserte par des naufragés, un cirque (pour l'enseignement précoce), etc. jusqu'à des simulations globales en français sur objectifs spécifiques.

61 On intégrera dans ce type de focalisation les approches fondées sur la réalisation de tâches (dont certaines versions prendront plutôt place dans une focalisation sur la cognition, cf. ci- dessous). Le Cadre européen commun de référence (Common European Framework) et les travaux de Rod Ellis, notamment, peuvent entrer dans ce cadre, sous certaines conditions (tâche orientée résultat, coopération entre apprenants, etc.).

On peut aussi classer dans cette catégorie les séquences d'actes de parole. Certes, elles sont souvent très décontextualisées, ce qui interdit $d$ 'y voir une « modélisation du monde extérieur ». Mais ces séquences (notamment sous la forme proposée par le Crapel) font la part des réussites et des échecs des actes de parole. De ce fait, on a déjà une petite modélisation.

On aura facilement remarqué que ce qui précède n'entre pas dans une « méthode » (sauf si l'on prend «méthode » au sens qu'a ce mot dans le Discours de la méthode de Descartes). Les méthodes ne peuvent, en effet, prendre en compte des activités pédagogiques de ce type. Tout au plus peuvent-elles les suggérer. C'est pourquoi ces activités pédagogiques demandent une formation d'enseignants qui soit adéquate. Il y a là un principe qu'il faut souligner: aucune méthode papier, numérique ou mixte ne peut justifier l'absence de formation en didactique.

\subsection{Focalisations thématiques sur un domaine}

Les domaines de focalisation thématique peuvent être très variés. C'est une question plus complexe qu'il n'y paraît. On peut lui apporter une réponse simple : focalisation sur la grammaire et sur l'apprentissage du vocabulaire. Une réponse plus élaborée sera: 
focalisation sur le domaine pragmatique. La réponse la plus complexe est: focalisation sur la cognition.

\subsubsection{Focalisation sur les domaines lexicaux et grammaticaux} faisant, elles ne se situent pas au niveau du rapport entre apprenants comme modélisation du monde (cf. ci-dessus), sauf dans certaines réalisations de jeux de rôle, car elles visent avant tout la constitution d'une compétence pragmatique chez l'apprenant, c'est-à-dire d'une capacité à élaborer des énoncés qui s'inscrivent dans un processus collaboratif conversationnel. Cela ne signifie pas que les approches communicatives respectent le principe de coopération de Grice : un « processus collaboratif » peut mettre en évidence des désaccords.

72 Les approches communicatives permettent de construire des scénarios interactifs qui mettent en évidence des comportements langagiers à acquérir. En cela, ils supposent chez l'apprenant une capacité à mémoriser certains de ces scénarios et à mettre en évidence des composantes réutilisables dans d'autres scénarios. Il y a donc, dans ce type de processus d'apprentissage, une question de granularité: quel est le grain d'activité discursive à la fois identifiable et recombinable avec d'autres dans de nouvelles stratégies de communication?

\subsubsection{Focalisation sur la cognition}

73 La notion d'approche cognitive n'est pas nouvelle (cf. Lugton \& Heinle, 1971) mais elle n'occupe une place importante que depuis la fin des années 1980. L'idée à la base d'une approche de type cognitif est qu'il faut travailler les représentations que se construit l'apprenant. La différence entre les deux grands types de didactique cognitive est le poids donné à l'interaction dans la construction de ces représentations.

Recherches en didactique des langues et des cultures, 6-2 | 2009 
La notion de didactique cognitive est assez récente eu égard à l'adjonction de cognitif à un item. La psychologie devient cognitive dans les années 1950-60 et est suivie par la neuropsychologie et la psychopathologie. La psychologie cognitive a succédé à la psychologie behavioriste. Contrairement à cette dernière qui se limitait aux observables (stimulus et réponse corrélée), elle étudie les états mentaux dans leurs aspects représentationnels et computationnels. Elle distingue les opérations de haut niveau qui traitent des séquences (phrases, relations transdiscursives) comme données et les opérations de bas niveau qui travaillent sur des micro-données (syllabes et mots notamment, mais aussi syntagmes du point de vue constructionnel), les rapports entre haut et bas niveaux étant relatifs (une lettre est le bas niveau d'un mot, un mot est le bas niveau d'une phrase).

Dans les activités en LE [langue étrangère] (y compris pour des sujets de niveau avancé), le degré d'attention porté aux différents aspects de l'activité n'est pas identique à ce qu'on peut observer en LM. On note, de manière générale, une plus grande focalisation de l'attention sur la réalisation de processus de "bas niveau" (aspects phonologiques, syntaxique...) par rapport aux aspects sémantiques de la tâche.

(Gaonac'h, 1990 : 43).

Une didactique cognitive repose sur un principe selon lequel comprendre un énoncé c'est être capable de produire une action et / ou de reformuler cet énoncé. C'est donc une conception fonctionnelle et non structurelle qui préside à cette orientation cognitive de la didactique.

76 La focalisation thématique sur la cognition est bien une focalisation sur un domaine et non sur un rapport : on travaille "ce qui se passe dans la tête de l'apprenant ». Dans ce cas, le travail grammatical doit porter à la fois sur l'exigence de mécanicité et sur la projection des séquences dans la stratégie discursive.

77 La notion d'énonciation utilisée en France dans la mouvance de Culioli par le groupe Charlirelle est proche de la notion de cognition et doit sans doute être traduite en anglais par cognition, le terme enunciation n'ayant pas pris en anglais la valeur prise par le terme « énonciation » en français.

\section{En guise de (non) conclusion}

Nous n'avons pas fourni un panorama des méthodologies auxquelles recourt la didactique des langues et nous n'en avions pas la prétention. Nous avons essayé de faire le pont entre la didactique et la linguistique en montrant que les questions génétiques en linguistique et la constitution d'objets à analyser étaient prioritaires pour l'établissement d'une relation.

\section{BIBLIOGRAPHIE}

Benveniste, É. (1966). Problèmes de linguistique générale, 1. Paris : Gallimard. 
Benveniste, É. (1974). Problèmes de linguistique générale, 2. Paris : Gallimard.

Besse, H. (1985). Méthodes et pratiques des manuels de langue. Paris : Didier.

Charlirelle (1975-1980). Behind the words. Paris : OCDL Hatier.

Conseil de l'Europe (2001). Cadre européen commun de référence pour les langues - Apprendre, enseigner, évaluer. Paris : Didier. Disponible en ligne : http://www.coe.int/fr/web/portfolio/thecommon-european-framework-of-reference-for-languages-learning-teaching-assessment-cefr-

Chomsky, N. (1977). Dialogues avec Mitsou Ronat. Paris : Flammarion.

Ellis, R. (2006). «The Methodology of Task-Based Teaching ». Asian EFL Journal, vol. 8, n 3. pp. 19-45.

Gaonac'h, D. (1990). «Les stratégies attentionnelles dans l'utilisation d'une langue étrangère ». Le français dans le monde, $n^{\circ}$ spécial « Acquisition et utilisation d'une langue étrangère: L'approche cognitive ». pp. 41-49.

Lugton, R. \& Heinle, J. (eds.) (1971). Toward a Cognitive Approach to Second Language Acquisition. Philadelphia, The Center for Curriculum Development.

Maingueneau, D. (1976). Initiation aux méthodes de l'analyse du discours - Problèmes et perspectives. Paris : Hachette.

Maingueneau, D. (1987). Nouvelles tendances en analyse du discours. Paris : Hachette.

O'Keefe, A., McCarthy, M. \& Carte, R. (2007). From Corpus to Classroom. Cambridge : Cambridge University Press.

Picoche, J. (1977). Précis de lexicologie française. Paris : Nathan.

Portine, H. (2005). « Le canard, de la désignation d'un oiseau à celle d'un journal ou l'homonymie peut-elle être cognitive ?". In C. Casseville \& Baudorre, P. (dir.). L'amitié, ce pur fleuve... Hommage à Bernard Cocula. Bordeaux : L'Esprit du Temps. pp. 95-116.

\section{NOTES}

1. Les syntagmes dans l'état actuel de la grammaire générative (minimalisme) sont un peu différents.

2. En fait, comme "opération", " analyse »" est polysémique, ce qui ne facilite pas l'intercompréhension. "Analyse" désigne d'abord le mouvement de décomposition d'un tout en parties mais "analyse" peut aussi désigner globalement le double mouvement d'analyse proprement dite et de synthèse. Cette occurrence $d$ '"analyse" correspond à ce deuxième sens, qui n'est finalement qu'une facilité de langage.

3. On remarquera que, là où l'on côtoie la grammaire traditionnelle, on oublie souvent dans les cursus de formation ce que l'on considère comme des évidences. La notion de fonction de notre grammaire scolaire est souvent traitée avec négligence parce qu'apparemment évidente (ou méprisée parce que traditionnelle) alors qu'il s'agit d'une notion fine et complexe par bien des aspects (comme l'identité fonctionnelle noyau / syntagme).

4. On trouvera deux conceptions différentes du lien entre «canard» au sens de journal et « canard » comme oiseau dans Picoche (1977: 76-79) et dans Portine (2005).

5. On n'a pas forcément conscience de ce fait. Un locuteur francophone ne considérera pas a priori que "chanter "est formé de deux parties, du fait qu'il a l'habitude d'exhiber cette forme comme pure manifestation du verbe "chanter". 
6. On a l'habitude d'appeler " proposition » une phrase dans une phrase mais ce n'est qu'un effet de contextualisation qui peut être pratique. Il en découle la distinction entre phrase simple et phrase complexe qui est un pur effet de théorisation et n'a donc aucune naturalité. On remarquera qu'il y a un parallélisme fonctionnel entre " mot », "syntagme » et "proposition » (exemple : nom, groupe nominal, complétive).

7. La lecture des sixièmes parties («Lexique » et «Culture ») de Benveniste $(1966,1974)$ reste encore d'actualité.

8. Le passage de linguistique à sciences du langage est un phénomène franco-français. On a vu que le passage de grammaire à linguistique a entraîné une augmentation considérable du territoire pris en compte dans les études. C'est ainsi qu'ont pu apparaître une sociolinguistique et une pragmatique, mais aussi une psycholinguistique. Une didactique des langues a aussi sa place en linguistique dès lors que l'on considère que « langues » est crucial dans le syntagme « didactique des langues", ce qui ne signifie pas pour autant que la didactique des langues doive se restreindre à une pure application de la linguistique mais qu'elle peut aussi faire appel à des disciplines complémentaires comme l'ethnologie, les sciences de l'éducation, etc. C'est en fait un problème de centrage. La didactique des langues pourrait aussi se centrer sur les sciences de l'éducation. Dans ce cas, «langues » perdrait de son acuité et la linguistique deviendrait une discipline complémentaire. Il faut choisir et c'est un choix sociopolitique. Mais comme, en France, la structure CNU (Conseil National des Universités) a pour conséquence des hiérarchies dans la représentation des disciplines, on a voulu éviter cette question en remplaçant « linguistique ", jugé trop lié au triplet < morphologie / syntaxe / sémantique >, par « sciences du langage ». C'est peine perdue. Il faut seulement accepter de ne pas être au centre de la linguistique et savoir justifier par le sérieux des connaissances et des études la reconnaissance des autres composantes de la linguistique, ou alors il faut entreprendre une course à l'infini dans la dénomination : « ensemble des sciences portant sur le langage », etc.

9. Rappelons que l'élève c'est le participant à une classe en tant que composante de l'institution. L'apprenant est un élève pris dans un processus d'apprentissage, ce à quoi l'élève ne passe pas tout son temps, par exemple lorsqu'il bavarde ou lorsque la classe est en train de négocier un calme suffisant pour que l'enseignant puisse faire son travail d'enseignant (cette négociation fait partie de son métier - à ne pas confondre avec travail - d'enseignant).

10. On pourrait faire le parallèle avec l'enseignement de l'histoire : une approche thématique y a eu notamment l'effet pervers suivant: Jules César et Napoléon avaient été mis dans certains cours sous le thème "conquête d'un pouvoir personnel en milieu républicain", ce qui peut se comprendre mais efface toute inscription dans un contexte donné. Dans notre domaine, on a vu des confusions entre méthode directe et Sgav parce que tous les deux tombaient sous le thème " utilisation exclusive de la langue étrangère ».

11. On a vu récemment des bandes entrer dans l'école pour des « règlements de compte » : ceci réinscrit la classe dans le monde sans le « rapport nouveau » en question mais de façon brutale.

12. Henri Besse (1985: 28-31) a distingué, avec beaucoup de justesse, la méthode "lecture traduction" de la méthode "grammaire - traduction». Nous préférons "méthodologie » à « méthode » mais sans accorder une importance très grande à cette question. 


\section{RÉSUMÉS}

L'auteur expose d'abord les objets et les questions qui animent la quête du linguiste. Puis il fournit une chronologie de la façon d'envisager la didactique des langues. Il termine en essayant de ponter positions en didactique et façons d'envisager la langue et l'activité langagière en recourant à la notion de focalisation thématique.

The author first sets out the subjects and the questioning which lead the quest of linguists. Then he supplies a chronology of the way how to view language teaching and learning. He ends trying to bridge positions on teaching and learning and the ways to view language and language skills resorting to the notion of thematic focusing.

\section{INDEX}

Mots-clés : approches communicatives, cognition, discours, énonciation, méthodologies, phrase, Sgav, tâches

Keywords : communicative approach, cognition, discourse, utterance, methodology, sentence, Sgav, task based learning

\section{AUTEUR}

\section{HENRI PORTINE}

Henri Portine, Telem (Telanco), université Bordeaux 3, France, a été chargé d'études au BELC et a enseigné à Paris 7 et à Lille 3 . Il est actuellement professeur à Bordeaux 3. Sa thèse de doctorat d'état (Paris 7, 1988, directeur : A. Culioli) portait sur le marqueur encore et ses valeurs temporelles, quantitatives et modales. En didactique des langues, il a publié sur l'argumentation et sur la didactique de la grammaire. En linguistique théorique, il a publié sur les temps verbaux, sur les modalités et sur la connexion. Il a participé à diverses expertises, a appartenu à la MSTP 6 et appartient actuellement au $\mathrm{CNU}-7^{\mathrm{e}}$ section.

Courriel : Henri.Portine[at]u-bordeaux3.fr

Adresse : UFR Lettres, Université Bordeaux 3, 33607 Pessac Cedex. 